\title{
Bacteraemia and antibiotic sensitivity in a tertiary neonatal intensive care unit
}

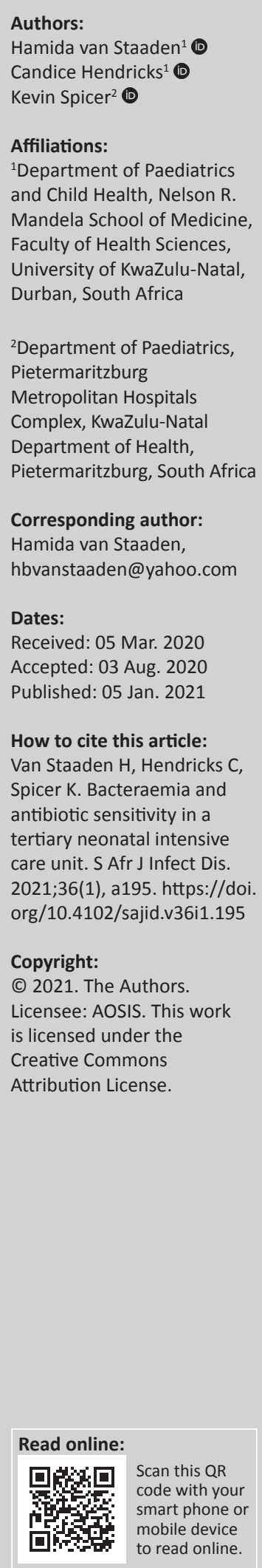

Background: Neonatal sepsis is an important cause of mortality and morbidity in neonatal intensive care populations worldwide. Data on rates of bacteraemia and antibiotic resistance patterns are limited, particularly in the developing world.

Methods: We retrospectively reviewed positive blood cultures obtained in the neonatal intensive care unit between 01 January 2015 and 31 December 2015. All neonates, either born at the tertiary hospital or transferred from referral units, regardless of diagnosis, who had a positive blood culture were included.

Results: There were 702 admissions during the study period and 437 positive cultures. Male patients made up 55.1\% (65/118), and the gender was unknown for $11.0 \%(13 / 118)$. Late onset sepsis accounted for $85.7 \%(102 / 119)$ and early onset sepsis, $14.3 \%(17 / 119)$. Of the 119 organisms cultured, 76 (63.8\%) were Gram-negative, 35 (29.4\%) were Gram-positive and 8 $(6.7 \%)$ were Candida species. Klebsiella was the most common genus at $42 \%(50 / 119)$. Of the clinically relevant organisms recovered, $37.0 \%$ (44/119) were susceptible to the empiric firstline regimen of penicillin and gentamycin. Furthermore, 69.7\% (53/76) of the Gram-negative organisms produced extended-spectrum beta-lactamases.

Conclusion: The majority of organisms cultured were considered contaminants and were not clinically relevant. Improvements in culture collection processes are needed. The majority of organisms considered clinically relevant were resistant to the first-line antibiotic regimen. To improve the likelihood of clinical success, empiric antibiotic regimens should be based on local data, if possible.

Keywords: neonatal sepsis; positive blood cultures; antimicrobial resistance; antibiotic resistance; culture collection processes.

\section{Introduction}

Neonatal sepsis is an important cause of mortality and morbidity in neonatal intensive care unit (NICU) populations worldwide. The World Health Organization reports that more than 4 million babies die in the first 28 days of life each year and that infections and sepsis, respectively, account for $36 \%$ and $6 \%$ of these neonatal deaths. ${ }^{1}$ Neonates, and particularly preterm newborns, are at higher risk of sepsis., ${ }^{2,3}$ Amongst the factors implicated are impaired cytokine production, reduced expression of adhesion molecules in neutrophils and reduced cytotoxic T-cell activity. ${ }^{4}$ Coupled with invasive interventions often carried out in the NICU, this leaves neonates at a considerably high risk for sepsis. ${ }^{4,5,6}$ Individual patient morbidity is not the only factor to consider. Neonates with suspected or proven sepsis require longer hospital stays, resulting in added cost and decreased turnover of beds. ${ }^{7,8}$ This is particularly problematic in the developing world with overcrowded nurseries. ${ }^{9}$ These pressures lead to liberal use of antibiotics and often rapid escalation to higher classes of drugs. ${ }^{9}$ Subsequently, organ dysfunction, ototoxicity and, more importantly, the development of antibiotic resistance become major factors to consider..$^{10,11}$

Diagnosis and early treatment of neonatal sepsis remain challenging, as the signs and symptoms of neonatal sepsis are often non-specific. ${ }^{9}$ Presentation varies and can include temperature instability, feeding difficulty, respiratory distress, lethargy and seizures. ${ }^{9,12}$ Prompt and efficient diagnosis and appropriate treatment are vital to prevent ongoing morbidity and mortality. Blood cultures are frequently performed in the NICU either on arrival to the unit or when there is clinical deterioration..$^{13}$ Blood cultures, despite lacking $100 \%$ sensitivity, remain the gold standard for confirmation of a diagnosis of septicaemia. ${ }^{4,14}$ Additionally, identification of the 
causative organism allows for antibiotic susceptibility testing and provides a basis for targeted, and more effective, antibiotic therapy. Organisms that are more commonly implicated in neonatal sepsis differ between developing and developed countries, and the former has been shown to have more healthcare-associated infections. ${ }^{15}$ Overall, Gram-negative organisms are more common and include Klebsiella species, Escherichia coli, Pseudomonas species and Salmonella species. ${ }^{2,7,15,16}$ Gram-positive organisms most commonly isolated include Staphylococcus aureus, coagulasenegative staphylococci (CONS) and Streptococcus pneumoniae..$^{2,15}$

A common practice is to start empiric antibiotics until sepsis is proven or excluded. ${ }^{13}$ This leads to widespread use of antibiotics as blood cultures often take days to identify an offending organism. Knowledge of the most common aetiological agents causing neonatal infection in a particular NICU is thus essential in developing strategies to prevent and treat serious neonatal infections in that unit. More effective prevention and treatment strategies will improve progress towards Millennium Development Goal 4, which targets mortality rates in children $<5$ years of age. ${ }^{17}$ Empirical treatment protocols are often formulated from data gathered in developed countries. The NICU at the tertiary hospital uses soluble penicillin and gentamycin as first-line empirical treatment for infants with suspected infection or sepsis. With increasing resistance to penicillin and gentamycin, the need for information on local antibiotic susceptibility patterns is crucial. ${ }^{3,5}$

The aim of this study was to determine the prevalence of positive blood cultures and describe the identified organisms and associated antibiotic susceptibility patterns in the NICU of a tertiary hospital. This will contribute towards the formulation of local empirical antibiotic regimens and will illustrate an approach that can be taken by other facilities.

\section{Methods}

This was a retrospective descriptive study, reviewing data from a tertiary NICU in KwaZulu-Natal, South Africa, for a 1-year period, from 01 January 2015 through 31 December 2015.

The NICU is a 26-bed tertiary unit that serves the neonatal population of Pietermaritzburg and referrals from Area 2 (comprising five districts with 20 hospitals) within KwaZulu-Natal, South Africa. The NICU consists of 6 beds for ventilation and 20 beds for neonatal high care. Care is provided to both medical and surgical patients.

All neonates, either born at the hospital or transferred from referral units, regardless of diagnosis, who had a positive blood culture were included in the study. Positive blood cultures were extracted from the National Health Laboratory Service (NHLS) computerised database. Information on patient demographics, dates when blood cultures were taken, organisms identified and antibiotic susceptibilities was collected. Data were entered into an Excel spreadsheet.

Patients were assessed for sepsis, and aseptic techniques were used to obtain blood cultures on admission as well as during periods of clinical deterioration. These episodes included, but were not limited to, periods of temperature instability, respiratory distress and need for ventilation and seizures. Blood volumes of $1 \mathrm{~mL}-2 \mathrm{~mL}$ were obtained from one site in each patient (unless a central line was present when two samples were considered at the discretion of the treating physician) and inoculated into one paediatric blood culture bottle (BACTEC Peds Plus Culture bottle). Samples were transported to the NHLS Microbiology Laboratory for incubation and were subsequently processed for susceptibility testing. The BACTEC instrument incubates and monitors the bottle for growth by detecting change in $\mathrm{pH}$ in the indicator at the bottom of the bottle. Changes in $\mathrm{pH}$ signal growth, and the bottle is flagged as positive. The positive blood culture bottle is then removed from the instrument, a Gram stain is made and the bottle contents are subcultured onto agar plates. The bottles remain in the instrument for 5 days after which they are removed if no growth occurs. The VITEK system (Vitek instrument for sensitivity testing, which uses the broth microdilution method) was used for organism identification and for ascertaining antibiotic susceptibility of recovered organisms.

Initiation of and changes in antibiotics were made by the attending paediatrician or neonatologist in the NICU. The antibiotic policy in the unit for infants with suspected infection or sepsis is as follows: first-line empirical therapy is soluble penicillin and gentamycin; second-line antibiotics include Tazocin (piperacillin-tazobactam) and amikacin, which have broader spectrum cover against most Gram-positive and Gram-negative aerobic and anaerobic bacteria, and third-line treatment is meropenem, which has the broadest range of cover against Gram-positive and Gram-negative bacteria. Antifungal therapy with fluconazole was included for premature neonates with persistent thrombocytopenia or who cultured positive for Candida species.

Neonatal sepsis was divided into early onset sepsis (EOS) and late onset sepsis (LOS). Early onset sepsis, defined for preterm infants as occurring within the first $72 \mathrm{~h}$ of life, is often attributed to acquisition of infection during the peripartum period. Late onset sepsis, occurring after $72 \mathrm{~h}$ of life, is often attributed to acquisition of infection in the NICU or in the community. ${ }^{10}$

\section{Study definitions}

- Contamination by commensals is a positive culture with the following organisms: corynebacterium species, micrococcus species, CONS, viridans group streptococci, propionibacterium species and bacillus species (Centers for Disease Control and Prevention definition). ${ }^{11}$ 
- Multidrug-resistant (MDR) is defined as an organism resistant to at least one agent in three or more antimicrobial categories. $^{18}$

- Extremely drug resistant (XDR) refers to an organism resistant to at least one agent in all but two or fewer antimicrobial categories. ${ }^{18}$

- Carbapenem-resistant organisms are usually discussed as a special subgroup of MDR organisms.

- Repeat isolates of the same organism within 14 days were considered most likely an infection that had not been appropriately or successfully treated. In these situations, it was considered the same event. These cultures were removed from analysis in the study ('non-significant cultures').

- Analyses were performed using SPSS software (Version 24.0). The $p$-values less than 0.05 were considered statistically significant.

\section{Ethical consideration}

Ethics approval was obtained from the Biomedical Research Ethics Committee of the University of KwaZulu-Natal (Ref: BE 452/16).

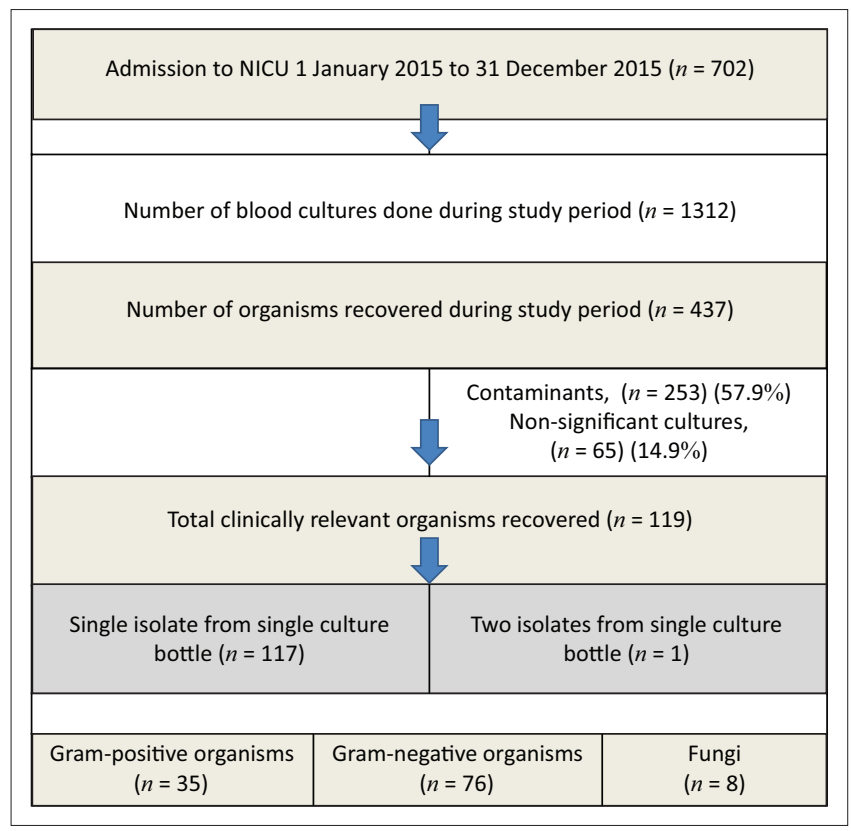

FIGURE 1: Study population.

\section{Results}

During this 1-year study period, there were 702 admissions to the NICU (Figure 1). The microbiology laboratory received 1312 blood cultures, and a total of 437 organisms were recovered. Of the organisms recovered, 253 were considered contamination by commensals and were not included in further analyses (CONS, $n=176$; bacillus species, $n=35$; enterococcus species, $n=17$; viridans group streptococci, $n=$ 12; micrococcus species, $n=7$; corynebacterium species, $n=1$; fungal species, $n=5$ ). A further 65 organisms were removed as they cultured positive within 14 days of a previous positive culture for the same organism ('non-significant cultures'). The 119 remaining organisms were obtained from 118 samples received (one infant had two organisms grown from a single blood culture bottle). The rate of clinically relevant blood culture positivity was thus $9.0 \%$ (118/1312). The rate of clinically relevant bacteraemia amongst the patients admitted to the NICU was $16.8 \%(118 / 702)$.

Gram-negative bacteria were the predominantly cultured organisms making up 63.8\% (76/119), with Gram-positive bacteria and fungi contributing 29.4\% (35/119) and 6.7\% $(8 / 119)$, respectively (Table 1$)$. Fungal infections were found significantly more in males $(p<0.01)$.

Regarding Gram-positive organisms, 74.3\% (26/35) were Staphylococcus species, 25.7\% (9/35) were Enterococcus species and of interest, no Streptococcus agalactiae (Group B streptococcus [GBS]) was seen in our cohort (Table 2). Amongst the Gram-negative bacteria, 65.8\% (50/76) were Klebsiella species and smaller percentages of patients had Serratia species $(13.2 \%, 10 / 76)$, Acinetobacter species $(7.9 \%$, 6/76), Enterobacter species $(3.9 \%, 3 / 76)$ and Escherichia coli $(3.9 \%, 3 / 76)$.

Included in Table 2 are data on Gram-positive organisms (Staphylococcus capitis, Staphylococcus epidermidis) that are often considered skin commensals. They were included here as they were recovered from more than one culture from the patient and were thus thought to be clinically relevant.

TABLE 1: Comparison of organisms in demographic groups.

\begin{tabular}{|c|c|c|c|c|c|c|c|c|}
\hline \multirow[t]{3}{*}{ Variable } & \multicolumn{6}{|c|}{ Organism recovered } & \multirow[t]{3}{*}{ Total patients } & \multirow[t]{3}{*}{$\%$} \\
\hline & \multicolumn{2}{|c|}{ Gram-positive bacterium } & \multicolumn{2}{|c|}{ Gram-negative bacterium } & \multicolumn{2}{|c|}{ Fungus } & & \\
\hline & $n$ & $\%$ & $n$ & $\%$ & $n$ & $\%$ & & \\
\hline \multicolumn{9}{|l|}{ Gender } \\
\hline Male & 20 & - & 38 & - & 7 & - & 65 & 54.6 \\
\hline Female & 12 & - & 28 & - & 1 & - & 41 & 34.4 \\
\hline Unknown & 3 & - & 10 & - & 0 & - & 13 & 10.9 \\
\hline \multicolumn{9}{|c|}{ Age (in days) } \\
\hline $0-3$ & 7 & - & 10 & - & 0 & - & 17 & 14.3 \\
\hline $4-7$ & 7 & - & 19 & - & 1 & - & 27 & 22.7 \\
\hline $8-28$ & 10 & - & 33 & - & 7 & - & 50 & 42.0 \\
\hline $28+$ & 11 & - & 14 & - & 0 & - & 25 & 21.0 \\
\hline Total & 35 & 29.4 & 76 & 63.8 & 8 & 6.7 & 119 & - \\
\hline
\end{tabular}

Note: The sex was unknown in $13(10.9 \%)$ patients and more positive cultures were obtained from males. Infants ranged in age from 0 to 84 days. The median age was 14 days. 
TABLE 2: Early onset versus late onset sepsis.

\begin{tabular}{|c|c|c|c|}
\hline \multirow[t]{2}{*}{ Organism subtype } & \multicolumn{2}{|c|}{$n$} & \multirow[t]{2}{*}{ Total } \\
\hline & EOS ( $\leq 3$ days old) & LOS (> 3 days old) & \\
\hline \multicolumn{4}{|l|}{ Gram-positive organisms ( $n=35)$} \\
\hline Staphylococcus species $(n=26)$ : & - & - & - \\
\hline Staphylococcus capitis & 0 & 1 & 1 \\
\hline Staphylococcus epidermidis & 2 & 8 & 10 \\
\hline Staphylococcus aureus & 3 & 12 & 15 \\
\hline Enterococcus species $(n=9)$ : & - & - & - \\
\hline Enterococcus faecium & 0 & 2 & 2 \\
\hline Enterococcus faecalis & 2 & 5 & 7 \\
\hline \multicolumn{4}{|l|}{ Gram-negative organisms ( $n=76)$} \\
\hline Klebsiella species $(n=50)$ : & - & - & - \\
\hline Klebsiella oxytoca & 0 & 4 & 4 \\
\hline Klebsiella pneumoniae & 7 & 39 & 46 \\
\hline Serratia marcescens $(n=10)$ & 0 & 10 & 10 \\
\hline Acinetobacter species $(n=6)$ : & - & - & - \\
\hline Acinetobacter Iwoffii & 1 & 1 & 2 \\
\hline Acinetobacter baumannii complex & 1 & 3 & 4 \\
\hline Enterobacter species $(n=3)$ : & - & - & - \\
\hline Enterobacter aerogenes & 0 & 1 & 1 \\
\hline Enterobacter cloacae & 0 & 2 & 2 \\
\hline Escherichia coli $(n=3)$ & 0 & 3 & 3 \\
\hline Stenotrophomonas maltophilia $(n=2)$ & 0 & 2 & 2 \\
\hline Neisseria subflava $(n=1)$ & 1 & 0 & 1 \\
\hline Proteus mirabilis $(n=1)$ & 0 & 1 & 1 \\
\hline \multicolumn{4}{|l|}{ Fungi $(n=8)$} \\
\hline Candida species: & - & - & - \\
\hline Candida parapsilosis & 0 & 1 & 1 \\
\hline Candida glabrata & 0 & 1 & 1 \\
\hline Candida guilliermondii & 0 & 1 & 1 \\
\hline Candida albicans & 0 & 5 & 5 \\
\hline Total & $17 \dagger$ & 102 & 119 \\
\hline
\end{tabular}

$\dagger, 14.3 \% ; \star 85.7 \%$.

EOS, early onset sepsis; LOS, late onset sepsis.

As noted in Table 2, most neonates had LOS (85.7\%, 102/119) as compared to EOS $(14.3 \%, 17 / 119)$. Klebsiella pneumoniae was the most common organism isolated in both EOS and LOS ( $n=7$ and $n=39$, respectively). All fungi cultured were Candida species and were included in the LOS category.

In Table 3, Staphylococcus species (other than Staphylococcus aureus) showed poor sensitivity to all antibiotics, except for vancomycin, to which all of the Gram-positive organisms were susceptible. Staphylococcus aureus was highly sensitive to trimethoprim-sulphamethoxazole (94\%), but minimally susceptible to clindamycin (13\%). Methicillin-resistant Staphylococcus aureus percentage was $80 \%(12 / 15)$.

Overall, 28.6\% (10/35) of the Gram-positive organisms cultured in this unit were susceptible to the first-line antibiotic regimen. Staphylococcus aureus was $26.7 \%$ susceptible, Staphylococcus species other than Staphylococcus aureus were 9.1\% susceptible and Enterococcus species were 55.6\% susceptible. Thus, identification of a Gram-positive organism would generally necessitate a change in antibiotic therapy.

Amongst the Gram-negative organisms, Klebsiella was the most frequently isolated genus and showed fairly good sensitivity to second-line antibiotics used in the unit, namely, piperacillin-tazobactam (78\%) and amikacin

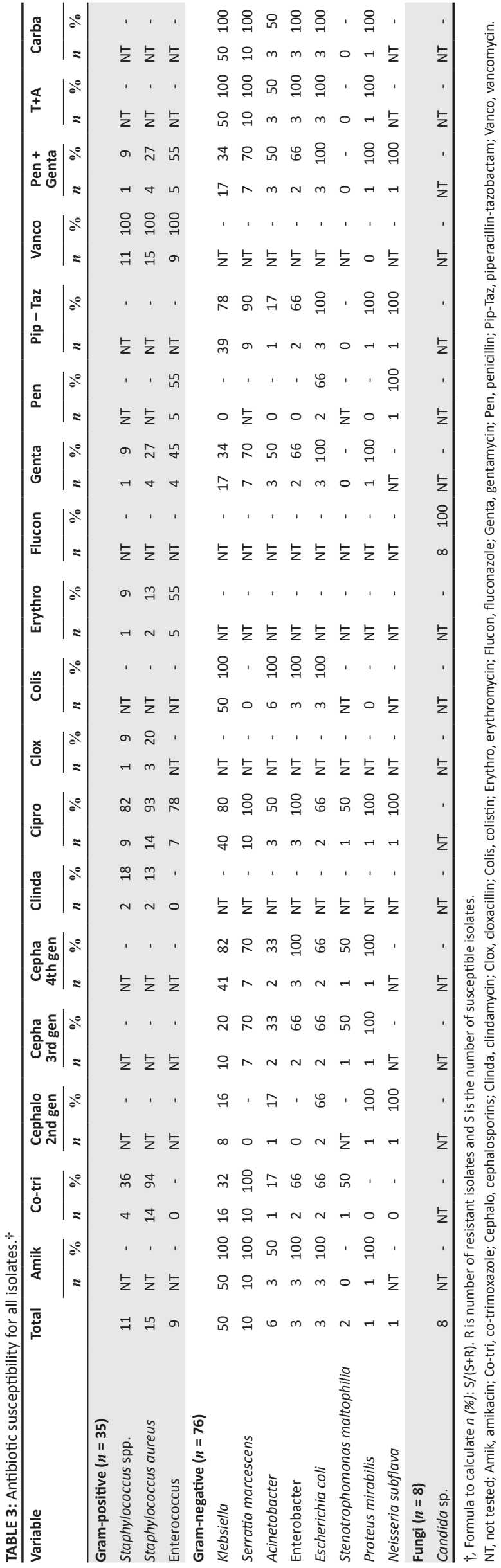


(100\%). Klebsiella species were consistently susceptible (100\%) to carbapenems (meropenem and imipenem) and showed high resistance to first-line empiric antibiotics (penicillin/ampicillin) ( $0 \%$ susceptible) and gentamycin (34\% susceptible). Of the 50 Klebsiella species isolated, 34 $(68 \%)$ were reported by the laboratory to produce an extended-spectrum beta-lactamase (ESBL).

Acinetobacter species showed high resistance to penicillin/ ampicillin ( $0 \%$ susceptible), piperacillin-tazobactam $(17 \%$ susceptible) and trimethoprim-sulphamethoxazole (17\% susceptible). Two (33\%) Acinetobacter baumannii were XDR, with resistance to all antibiotics except colistin. There were only three Escherichia coli isolated, and despite one ESBL producer, all were susceptible to first-line antibiotics. Enterobacter species showed high susceptibilities (> 80\%) to all agents except penicillin/ampicillin $(0 \%)$.

Overall, 44.7\% (34/76) of the Gram-negative organisms cultured in the unit were susceptible to the first-line antibiotics, whilst most were susceptible to second-line ( $93.4 \%$ susceptible) and third-line (93.4\% susceptible) antibiotics.

The Candida species isolated were all highly sensitive to fluconazole $(100 \%)$.

\section{Discussion}

During this 1-year study period, the incidence of cultureproven, clinically relevant neonatal bacteraemia was $16.8 \%$ $(118 / 702)$. The rate of clinically relevant culture positivity was $8.9 \%$ (118/1312). Studies by Kayange et al. from Tanzania and Pokhrel et al. in Nepal found clinically relevant neonatal bacteraemia incidence rates higher at 39\% and 20.5\%, respectively. ${ }^{9,19}$ Local South African data, however, provided incidences of $8.5 \%$ by Motara et al. in Johannesburg and $11.5 \%$ by Morkel et al. in Cape Town. ${ }^{16,20}$ Variability in culture positivity exists between units. This may be attributed to differences in culture technique or administration of antibiotics prior to cultures being drawn. Gram-negative organisms were the most frequently isolated organisms in our study, similar to what has been found in many studies from developing countries. ${ }^{16,19}$

The timing of positive cultures also plays a role in identifying possible causative factors. Early onset sepsis is associated with organisms found in the female genital tract, often GBS and E. coli. ${ }^{9}$ Group B streptococcus was not isolated in our study. This could be attributed to intrapartum administration of antibiotics for suspected and proven premature rupture of membranes. Escherichia coli was also not cultured in EOS. Late onset sepsis was more common in our NICU, which differs from findings in other studies and raises concerns regarding adherence to infection prevention and control measures in our unit., ${ }^{5,14}$ Adherence to infection prevention and control practices can be negatively affected by rapid turnover of beds and staff shortages, as well as other unit-specific conditions. These will need to be considered, and improvements will likely require a holistic approach to establish protocols targeting improved infection-control practices.

Coagulase-negative staphylococci are usually skin commensals and are often of uncertain clinical significance. Of the 437 organisms recovered from blood cultures in the present study, 176 (40\%) were CONS, which is consistent with results from other centres. Coagulase-negative staphylococci were found in $40 \%$ of positive cultures by Giannoni et al. in Switzerland and in $56 \%$ of positive blood cultures in a local South African study by Lebea et al. ${ }^{21,22}$ The increase in CONS could be due to poor blood culture sampling technique, but the possibility of clusters of CONS infections must also be considered. The latter may be due to the increasing numbers of very-low birth weight babies being cared for in the NICU, making septicaemia with normal skin commensal flora more commonplace. ${ }^{4} \mathrm{~A}$ more in-depth look at the results regarding timing of cultures and clinical correlates would have to be undertaken to try and determine factors influencing these trends. ${ }^{10}$ Educating members of the healthcare team on proper blood culture collection is vital in improving the quality of samples taken and ultimately the correct care of patients with positive blood cultures..$^{15,23}$

With antibiotic resistance being a topic of global concern, knowledge of local antibiotic resistance patterns is vitally important. ${ }^{7}$ Our study showed that the majority of the Grampositive organisms grown were resistant to first-line antibiotics: penicillin and gentamycin. Local data as well as data from India and Nepal showed complete resistance of Staphylococcus species to penicillin and gentamycin. ${ }^{19,20,23}$ These findings suggest that the first-line antibiotic policies may require revision to enable more sufficient coverage of the organisms present in this specific NICU.

Analysis of cultures obtained aid with guidelines for our unit. In this study, EOS was mostly caused by Gram-negative organisms, namely, Klebsiella species. There were no GBS. When CONS are grown, the use of the patient's clinical picture as well as the use of septic markers should guide the use of antibiotics. A second culture positive for CONS would also suggest that the organism is not a skin commensal in that particular clinical situation. The final choice of antibiotics should be guided by the particular organism grown and its sensitivity pattern. A large majority of our patients had LOS caused by an array of Gram-negative organisms. More concerning is the number and degree of MDR organisms cultured, the large number of ESBL-producing Klebsiella species, as well as the presence of XDR Acinetobacter baumannii. A majority of these cultures form a part of the LOS category. This serves as motivation for the formation of an infection control team to make regular visits to the unit for review of positive cultures and to reinforce infection control measures in the unit.

There are several limitations to this study. As it was a laboratory audit, clinical parameters such as birth weight, 
presence of central lines and outcomes of patients were not known. The determination of whether certain recovered organisms (especially the frequent CONS species in our study) were clinically significant was therefore not possible. Parameters such as gestational age, presence of central lines and data on occupation rates and staff ratio per bed would have further aided the analysis and should be included in future studies. This would allow for better elucidation of the reasons for the high number of positive cultures.

A further limitation is that the data are presently 4 years old, and certainly organisms and antibiotic sensitivities may have changed in the interim. The authors do however believe it still to be relevant as practices in the mentioned NICU have changed subsequently to better address infection control. The year following the collection of these samples, a new NICU was commissioned and a Healthcare Associated Infection Quality Improvement Team was formed. The work of this team included ward rounds with the infection control nurse, microbiologist and paediatric infectious diseases specialist. The team advised the clinicians on antibiotic choice and appropriate antimicrobial interventions. The newly built NICU helped with bedspacing and improved occasional overcrowding in the unit. An audit after the initiation of these measures would certainly aid to evaluate the impact of the interventions. Furthermore, analysis of data over the course of 2-5 years may assist in identifying the spectrum of cultured organisms and antibiotic susceptibility patterns. This information may thus better guide the formulation of local empirical antibiotic regimens.

\section{Conclusion}

Although limited by the lack of clinical data, this study does provide evidence of high numbers of a broad array of Gram-positive and Gram-negative pathogens responsible for neonatal bacteraemia. Problems with infection control in the unit are suggested by substantial levels of antimicrobial resistance, with only $39.6 \%(44 / 111)$ of the bacteria recovered susceptible to the first-line antibiotic regimen, as well as the predominance of LOS, a marker of nosocomial infection. Subsequent measures to improve infection control have been implemented, such as regular ward rounds with an infection control team, implementation of improved antibiotic policies and improved bedspacing in a new unit. An audit to establish the impact of these measures, whilst including important clinical information to provide context and significance, would be an important step to determine progress.

\section{Acknowledgements}

The authors would like to thank Dr Haffejee from National Health Laboratory Service, who assisted with collection of data and provided guidance on earlier drafts of this article, and Dr W. Sibanda from the College of Health Sciences, University of KwaZulu-Natal, for assisting with statistics and analysis in earlier drafts.

\section{Competing interests}

The authors declare that they have no financial or personal relationships that may have inappropriately influenced them in writing the manuscript.

\section{Authors' contributions}

H.v.S. was the primary author and analysed the data. C.H. and K.S. supervised the study and reviewed the article.

\section{Funding information}

This research received no specific grant from any funding agency in the public, commercial or not-for-profit sectors.

\section{Data availability statement}

Data sharing is not applicable to this article as no new data were created or analysed in this study.

\section{Disclaimer}

The views and opinions expressed in this article are those of the authors and do not necessarily reflect official policy or position of any affiliated agency of the authors.

\section{References}

1. World Health Organization. World health report 2005: Make every mother and child count. Geneva: World Health; 2005.

2. Gkentzi D, Kortsalioudaki C, Cailes BC, et al. Epidemiology of infections and antimicrobial use in Greek Neonatal Units. Arch Dis Child - Fetal Neonatal Ed [serial online]. 2019 May;104(3):F293-F297. Available from: http://fn.bmj.com/ lookup/doi/10.1136/archdischild-2018-315024

3. Wynn JL. Defining neonatal sepsis. Curr Opin Pediatr [serial online]. 2016 Apr;28(2):135-140. Available from: https://journals.Iww.com/co-pediatrics/ Fulltext/2016/04000/Defining_neonatal_sepsis.3.aspx

4. Zea-Vera A, Ochoa TJ. Challenges in the diagnosis and management of neonatal sepsis. J Trop Pediatr [serial online]. 2015 Feb 1;61(1):1-13. Available from: https://academic.oup.com/tropej/article-lookup/doi/10.1093/tropej/fmu079

5. Doronjski A, Barišić $\mathrm{N}$, Stojanović $\mathrm{V}$. Risk factors for neonatal sepsis and method for reduction of blood culture contamination. Malawi Med J [serial online]. 2015 Apr 24;27(1):20. Available from: http://www.ajol.info/index.php/mmj/article/ view/116232

6. Shane AL, Sánchez PJ, Stoll BJ. Neonatal sepsis. Lancet [serial online]. 2017 Oct;390(10104):1770-1780. Available from: https://linkinghub.elsevier.com/ retrieve/pii/S0140673617310024

7. Tsai M-H, Chu S-M, Hsu J-F, et al. Risk factors and outcomes for multidrug-resistant gram-negative bacteremia in the NICU. Pediatrics [serial online]. $2014 \mathrm{Feb}$ 1;133(2):e322-e329. Available from: http://pediatrics.aappublications.org/cgi/ doi/10.1542/peds.2013-1248

8. McCarthy K, Hawke A, Dempsey E. Antimicrobial stewardship in the neonatal unit reduces antibiotic exposure. Acta Paediatr [serial online]. 2018 Oct;107(10): 1716-1721. Available from: http://doi.wiley.com/10.1111/apa.14337

9. Kayange N, Kamugisha E, Mwizamholya DL, Jeremiah S, Mshana SE. Predictors of positive blood culture and deaths among neonates with suspected neonatal positive blood culture and deaths among neonates with suspected neonatal
sepsis in a tertiary hospital, Mwanza- Tanzania. BMC Pediatr [serial online]. 2010 sepsis in a tertiary hospital, Mwanza- Tanzania. BMC Pediatr [serial online]. 2010
Dec 4;10(1):39. Available from: http://bmcpediatr.biomedcentral.com/ Dec 4;10(1):39. Available from: http://bmcpediatr.biomedcentral.com/
articles/10.1186/1471-2431-10-39

10. Shrestha RK, Rai SK, Khanal LK, Manda PK. Bacteriological study of neonatal sepsis and antibiotic susceptibility pattern of isolates in Kathmandu, Nepal. Nepal Med Coll J. 2013 Mar;15(1):71-73.

11. Centers for Disease Control and Prevention. National Healthcare Safety Network (NHSN) Overview [homepage on the Internet]. 2020. Available from: https:// www.cdc.gov/nhsn/PDFs/pscManual/1PSC_OverviewCurrent.pdf

12. Perera KSY, Weerasekera M, Weerasinghe UDTM. Risk factors for early neonatal sepsis in the term baby. Sri Lanka J Child Heal [serial online]. 2018 Mar 5:47(1):44 Available from: https://sljch.sljol.info/article/10.4038/sljch.v47i1.8429/

13. Ntusi N, Aubin L, Oliver $S$, et al. Guideline for the optimal use of blood cultures. SAMJ S Afr Med J. 2010;100(12):839-843. https://doi.org/10.7196/SAMJ.4217

14. Galhotra S, Gupta V, Bains H, Chhina D. Clinico-bacteriological profile of neonatal septicemia in a tertiary care hospital. J Mahatma Gandhi Inst Med Sci [serial online]. 2015;20(2):148. Available from: http://www.jmgims.co.in/text. asp?2015/20/2/148/164240 
15. Vergnano S. Neonatal sepsis: An international perspective. Arch Dis Child - Fetal Neonatal Ed [serial online]. 2005 May 1;90(3):F220-F224. Available from: www. archdischild.com

16. Motara F, Ballot DE, Perovic O. Epidemiology of neonatal sepsis at Johannesburg Hospital. S Afr J Epidemiol Infect [serial online]. 2005 Jan 15;20(3):90-93. Available from: https://www.tandfonline.com/doi/full/10.1080/10158782.2005.11441243

17. United Nations. The millennium development goals report [homepage on the Internet]. United Nations, 2015; p. 72. Available from: https://visit.un.org/ millenniumgoals/2008highlevel/pdf/MDG_Report_2008_Addendum.pdf

18. Magiorakos A-P, Srinivasan A, Carey RB, et al. Multidrug-resistant, extensively drug-resistant and pandrug-resistant bacteria: An international expert proposa for interim standard definitions for acquired resistance. Clin Microbiol Infect [serial online]. 2012 Mar;18(3):268-281. Available from: https://linkinghub. elsevier.com/retrieve/pii/S1198743X14616323

19. Pokhrel B, Koirala T, Shah G, Joshi S, Baral P. Bacteriological profile and antibiotic susceptibility of neonatal sepsis in neonatal intensive care unit of a tertiary hospita in Nepal. BMC Pediatr [serial online]. 2018 Dec 27;18(1):208. Available from: https://bmcpediatr.biomedcentral.com/articles/10.1186/s12887-018-1176-x
20. Morkel G, Bekker A, Marais BJ, Kirsten G, van Wyk J, Dramowski A. Bloodstream infections and antimicrobial resistance patterns in a South African neonatal intensive care unit. Paediatr Int Child Health [serial online]. 2014 May 6;34(2): 108-114. Available from: http://www.tandfonline.com/doi/full/10.1179/204690 108-114. Available

21. Giannoni E, Agyeman PKA, Stocker M, et al. Neonatal sepsis of early onset, and hospital-acquired and community-acquired late onset: A prospective population-based cohort study. J Pediatr [serial online]. 2018 Oct;201: 106-114.e4. Available from: https://linkinghub.elsevier.com/retrieve/pii/ S0022347618307613

22. Lebea MM, Davies V. Evaluation of culture-proven neonatal sepsis at a tertiary care hospital in Johannesburg, South Africa. SAJCH South African J Child Health 2017;11(4):170-173.

23. Sharma CM. 'Neonatal sepsis': Bacteria \&amp; their susceptibility pattern towards antibiotics in neonatal intensive care unit. J Clin Diagnostic Res [serial online] 2013;7(11):2511-2513. Available from: http://www.jcdr.net/article_fulltext. asp?issn=0973-709x\&year=2013\&volume $=7 \&$ issue $=11 \&$ page $=2511 \&$ issn $=0973$ $709 \times \& i d=3594$ 